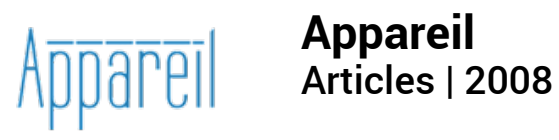

\title{
Augustin et Tolstoï : confession, littérature, philosophie
}

\section{Mehmet Aydin}

\section{Q OpenEdition}

Journals

Édition électronique

URL : http://journals.openedition.org/appareil/282

DOI : 10.4000/appareil.282

ISSN : 2101-0714

Éditeur

MSH Paris Nord

\section{Référence électronique}

Mehmet Aydin, « Augustin et Tolstoï : confession, littérature, philosophie », Appareil [En ligne], Articles, mis en ligne le 15 mars 2008, consulté le 30 juillet 2020. URL : http://journals.openedition.org/ appareil/282 ; DOI : https://doi.org/10.4000/appareil.282

Ce document a été généré automatiquement le 30 juillet 2020.

\section{(c) (i) (9)}

Appareil est mis à disposition selon les termes de la Licence Creative Commons Attribution - Pas d'Utilisation Commerciale - Pas de Modification 4.0 International. 


\title{
Augustin et Tolstoï : confession, littérature, philosophie
}

\author{
Mehmet Aydin
}

1 Romancier, conteur, essayiste, pédagogue, prédicateur, penseur politique et religieux, sociologue, philosophe, Tolstoï est une des figures les plus marquantes de la littérature russe et mondiale. Le message universel de son œuvre se présente sous des facettes multiples. Quel est le principe d'unification de cette multiplicité ? Y a-t-il une hypothèse explicative rendant raison du sens de la profusion créatrice et de la singularité de ce penseur atypique? Quel est le fil conducteur qui mène de l'homme à ses œuvres et de ses œuvres à sa vie? Notre propos ne sera donc ni littéraire, ni artistique, ni même à proprement parler religieux, mais philosophique ${ }^{1}$. C'est à la frontière de toutes ces disciplines que la philosophie entendue comme exercice nous permettra de circuler afin de mettre en évidence l'aspect vivant de cet exercice, tel qu'il est pratiqué aussi par Tolstoï. Pour l'écrivain russe, l'expérience vécue ne sera jamais remplacée par le concept, au sujet vivant on ne pourra jamais substituer une opération intellectuelle. Au vrai, quel rapport la philosophie entretien-elle avec la littérature ? Y a-t-il une forme d'expression proprement philosophique? La question n'est pas neuve. Nietzsche ne nous avait-il pas déjà averti ?

«Grande perplexité : la philosophie est-elle un Art ou une Science? C'est un Art dans ses fins et dans ses produits. Mais son moyen d'expression, l'exposition par concepts, lui est commun avec la science. C'est une de la poésie. Impossible de la classer. Il nous faudra inventer et caractériser une catégorie nouvelle... ${ }^{2}$

On sait que le roman suscite des réflexions philosophiques. V. Descombes ${ }^{3}$, en analysant l'univers romanesque de Proust (A la recherche du temps perdu), avance son hypothèse : le roman proustien délivre un type d'analyse qui est propre au roman qui le distingue de l'analyse philosophique. Si le souci du vrai est commun au roman et à la philosophie, leur forme d'investigation est différente. Rappelons brièvement que, les philosophes tels que G. Deleuze et J. Derrida ont écrits sur le sujet.

Dans notre problématique, le style confessionnel apparait la clef de l'originalité de l'œuvre tolstoïenne. Pour Thomas Mann : «Les œuvres littéraires de Tolstoï ne sont au fond que les fragments d'un immense journal tenu pendant cinquante années, une 
confession détaillée et sans fin. $»^{4}$ La confession comme un exercice philosophique original, se manifeste tout au long de la vie de l'auteur et dans toutes ses œuvres sous différentes formes.

4 En effet, la confession est en soi un genre littéraire et comportemental (éthique) codifié, qui a une histoire propre. Ses origines sont complexes; son rapport à l'ensemble de la philosophie est problématique. Selon nous, l'hypothèse selon laquelle ce concept historiquement surdéterminé prend un sens renouvelé et fondamental avec Tolstoï, en expliquent au préalable les caractéristiques généalogiques de la confession. En effet, le dire confessionnel traverse l'histoire de la culture occidentale. Il est intimement lié à la religion chrétienne, la confession prend également racine dans la philosophie antique. Au premier abord la confession est une institution religieuse bien précise. Le dictionnaire de la foi catholique la définit ainsi :

«Le double sens du mot latin est celui d'aveu, mais aussi de manifestation publique de reconnaissance. La confession est l'un des éléments du sacrement de pénitence, qui désigne souvent dans la langue courante le sacrement lui-même. La confession des péchés est la reconnaissance devant Dieu et devant son Eglise (représentée par son ministre) de son état de pécheur, non pas seulement en général (ce qui n'est pas difficile), mais de façon précise, en détaillant en quoi on a été pécheur. $»^{5}$

5 Dans le catholicisme, pour le croyant, par la conscience de son péché propre et la culpabilité qui s'ensuit, ses désirs et les passions du corps seront vus comme l'aveu de la chair, en vue d'une expiation nécessaire par la pratique confessionnelle. Selon Foucault :

«L'extension de l'aveu, et de l'aveu de la chair, ne cesse de croître. Parce que la Contre-Réforme s'emploie dans tous les pays catholiques à accélérer le rythme de la confession annuelle. Parce qu'elle essaie d'imposer des règles méticuleuses d'examen de soi-même. Mais surtout parce qu'elle accorde de plus en plus d'importance dans la pénitence - et aux dépens, peut-être, de certains autres péchés - à toutes les insinuations de la chair: pensées, désirs, imaginations voluptueuses, délectations, mouvements conjoints de l'âme et du corps, tout cela désormais doit entrer, et en détail, dans le jeu de la confession et de la direction. ${ }^{6}$ « Faire avouer le pécheur pour qu'il reçoive du prêtre le pardon divin et s'en aller rassuré : telle a été l'ambition de l'Eglise catholique, surtout à partir du moment où elle rendit obligatoire la confession privée chaque année et demande en outre aux fidèles l'aveu détaillé de tous leurs péchés "mortels". »"

Et «Toutes les chronologies destinées aux élèves de l'enseignement devraient donner un fort relief à la décision du concile de Latran IV (1215) qui rendit la confession annuelle obligatoire. La généralisation de cette contrainte, déjà en vigueur auparavant dans plusieurs diocèses, modifia la vie religieuse et psychologie des hommes et femmes de l'Occident, et pesa énormément sur les mentalités jusqu'à la Réforme dans les pays protestants et jusqu'au $\mathrm{XX}^{\mathrm{e}}$ siècle dans ceux qui demeurèrent catholiques. $\rrbracket^{8}$

6 Parallèlement à cette confession institutionnalisée, qui implique l'aveu et le pardon par l'intermédiaire d'un ministre de Dieu incarné par le prêtre, il existe une confession constituée en exercice philosophico-religieuse qui s'effectue sans la médiation d'une église ou d'une autorité religieuse intervenant pour recueillir l'aveu du confesseur.

Dans la philosophie occidentale, le style confessionnel a ses figures tutélaires : SaintAugustin, Rousseau, Nietzsche, Kierkegaard, Tolstoï. Une histoire de deux mille ans relie ces penseurs que tout sépare et que le dire confessionnel s'apparente. De plus, la confession traverse les concepts fondateurs de la modernité : l'individu, ses rapports à l'Histoire linéaire et finalisée, ainsi qu'à la mort. La confession dans ses relations 
contradictoires avec la philosophie identifierait l'Occident. Saint-Augustin, en tant que l'auteur d'un texte, devenu le modèle du genre, Confessions, et un des pères fondateurs de l'Eglise catholique, peut être considéré comme représentant un moment crucial de ce processus d'identification. D'autant qu'il se situe à cheval sur la confession comme institution religieuse non encore codifié et la confession comme exercice philosophicoreligieuse. Par delà l'abîme historique qui sépare les deux penseurs, Saint-Augustin est un passage obligé pour comprendre la démarche tolstoïenne.

8 Auteur du $\mathrm{V}^{\mathrm{e}}$ siècle de notre ère Augustin se relie à la tradition des penseurs grecs antiques dont Platon et Plotin. Il présente toujours une originalité pour les modernes, notamment par sa réflexion sur le temps, sur l'exploration existentielle de la temporalité et de la condition humaine. Son influence a été immense dans la formation de l'identité occidentale. Jean-Claude Eslin, dans Saint Augustin L'homme occidental, souligne son importance et son actualité, en nous invitant à réfléchir sur lui : Augustin est le premier moderne. Par rapport à l'empire romain et par rapport au christianisme d'Orient, il marque une rupture et représente le moment fondateur de l'inquiétude occidentale. Ainsi, il a inauguré une instabilité féconde, créatrice. Un homme qui dit $J e$ dans les Confessions et Nous dans La Cité de Dieu :

« Si, à nos yeux, Augustin est l'homme occidental, bon gré, mal gré nous sommes ses héritiers. Alors même que nous nous révoltons contre lui, ce que nous faisons depuis trois siècles, où que nous feignions de l'ignorer, nous ne pouvons jamais le refuser totalement car il nous a formés. Travailler avec lui, c'est donc travailler sur nous-mêmes. C'est pourquoi nous l'interrogeons ici sur ce qu'il a formé pour nous, la philosophie, l'homme, la société. ${ }^{9}$

9 L'influence d'Augustin, en tant que figure fondatrice, a été immense, même si l'on ne pouvait pas voir dans l'augustinisme ni tout le christianisme, ni tout le christianisme occidental dont il ne représente qu'une branche catholique particulière :

«La civilisation du christianisme oriental n'a pas connu Augustin, et ne cherche pas à le connaître. Son influence ne s'est pas exercée dans le domaine oriental de l'Empire, ni plus tard en Europe orientale, chez les Russes. Les problématiques qu'il a rendues familières à l'Occident sont là-bas restées étrangères. Ceci est important pour comprendre les différences de mentalités entre l'Ouest et l'Est de l'Europe. L'Orient chrétien est demeuré unitif et "synergique". Il ne fait pas éclater les contradictions, ce qui est par excellence la méthode d'Augustin. $»^{10}$

10 Pour K. Jaspers, Saint Augustin est la grande figure qui représente le mieux la pensée chrétienne. Sa pensée est capitale pour comprendre les origines de la pensée occidentale moderne. Son influence a laissé une longue continuité qui commence avec ses Confessions comme exercice philosophique d'une "critique dénonciatrice de soi ». Dans cette perspective, K. Jaspers met en parallèle Augustin avec Nietzsche et Kierkegaard, pour souligner la portée de l'influence et «la "modernité" de Saint Augustin» :

"Cette critique dénonciatrice de soi-même se poursuit à travers les penseurs chrétiens, jusqu'à Pascal, jusqu'à Kierkegaard et jusqu'à Nietzsche. " ${ }^{11}$ "Pour nous et notre temps, écrivait K. Jaspers, une comparaison de Saint Augustin avec Kierkegaard et Nietzsche est riche d'enseignement. (...). Saint Augustin compose la première véritable autobiographie et conclut son ouvrage par un coup d'œil rétrospectif qui est un examen critique de soi (comme l'ont fait Kierkegaard et Nietzsche). Ils apportent au lecteur, non seulement l'œuvre, mais l'idée qu'ils s'en font en réfléchissant à son sens. Comme, pour tous les trois, l'œuvre est devenue manifestation subjective, le portrait de chacun d'eux par lui-même y est parfaitement à sa place. Toutes les analogies entre ces penseurs attestent la 
profondeur d'émotion, l'aptitude aux expériences extrêmes, la puissante personnalité, la "modernité" de saint Augustin. (... ). L'état d'esprit qui règne dans tous ses écrits n'est pas celui qu'on trouve chez les grands éveilleurs Nietzsche et Kierkegaard: dans toute sa passion demeure le sens de la mesure et de la responsabilité. (...). Augustin jette des bases, il appartient à une puissance temporelle, il est au service de l'Eglise. (...). Kierkegaard, lui, se dresse seul en face de l'Eglise, "un mouchard au service de Dieu", ainsi qu'il se nomme lui-même. Nietzsche est seul, sans Dieu, questionnant et mis en question à l'infini, cherchant appui en vain à travers "l'éternel retour", la "volonté de puissance", la "vie dionysiaque". $»^{12}$

11 Les Confessions ont inauguré un type de réflexion sur le sens de l'existence humaine dont l'importance et l'actualité ne se démentent pas, comme en témoigne l'influence qu'elles ont exercé sur la philosophie du XXe siècle : Husserl, Heidegger, Wittgenstein, Arendt, Foucault, Derrida et Ricœur... Son œuvre a nourrit de nombreuses réflexions philosophiques jusqu'à nos jours. Ainsi, P. Ricoeur, en voulant concilier son travail philosophique et sa conviction religieuse, avoue-t-il, dans La Critique et la conviction : " Augustin a toujours joui, à mes yeux, d'une sorte de préférence... » ${ }^{13}$ Les Confessions peuvent toujours interpeller et accompagner l'homme moderne occidental dans sa quête. Pour J.-F. Lyotard, dans son œuvre inachevée La confession d'Augustin :

«De qui les Confessions sont-elle l'ouvrage, l'opus? Et pour le dire autrement, qu'œuvrent-elles, que mettent-elles en œuvre ou à l'œuvre, qu'ouvrent-elles, à quoi ouvrent-elles l'œuvre? L'ouverture donne le ton. Et ce ton est un leitmotiv, un fil conducteur qui ne cesse de tenir mon ton à l'ordre de ton omniscience. L'introït de l'œuvre à ta présence, et cette invocatio, la voix par laquelle j'appelle ta voix à venir parler dans ma voix, se répète au long des treize livres, ma voix se rappelle à ta voix, en appelle à elle, comme un refrain. Mon œuvre de confession, de narration et de méditation, n'est mon œuvre que parce qu'elle est la tienne. $»^{14}$

Rappelons que l'essai confessionnel non achevé de J.-F. Lyotard est interrompu par la mort. Il présente à peine la moitié du volume projeté.

13 J.Guitton, dans son essai intitulé Actualité de Saint Augustin, propose quelques orientations de lecture pour chercher l'influence d'Augustin sur les penseurs et écrivains contemporains, en commençant par Freud, jusqu'à Proust, Gide, Sartre :

«Je voudrais tenter de répondre, en envisageant sans cesse l'actualité de Saint

Augustin, et la ressemblance de son temps avec le nôtre. $»^{15}$

Beaucoup s'inscriront dans le sillage de l'autobiographie augustinienne. Les Confessions ont été l'un des livres clefs de l'Occident, comme le montre l'enquête de P. Courcelle : Les "Confessions " de saint Augustin dans la tradition littéraire ${ }^{16}$ nous donne une première idée sur l'influence d'Augustin dans la littérature et la philosophie: Dante, Pétrarque, Saint François de Sale, Bérulle, Pascal, Fénelon, Rousseau, Chateaubriand, Sainte-Beuve, Renan, Kierkegaard, Nietzsche...

15 Dans la pensée d'Augustin, nous trouvons la vocation universaliste finement élaborée de la chrétienté. La présence de Dieu se lit dans une humanité qui chemine depuis le jardin d'Eden jusqu'au jugement dernier. Humanité est entre deux amours, entre deux cités. La lecture de La Cité de Dieu, comme une œuvre fondatrice dans la pensée chrétienne, peut toujours nous inciter à réfléchir sur les liens supposés entre l'histoire, progrès et salut chrétien, et sur la modernité occidentale. Selon G. Vattimo :

«Si le progrès devient routine, c'est aussi parce que le développement de la technique a été préparé et accompagné, sur le plan théorique, par la "sécularisation" de la notion même de progrès; à travers un processus que l'on pourrait décrire comme le déroulement logique d'un raisonnement, l'histoire des 
idées a conduit à l'évidemment du concept de progrès. L'histoire, qui apparaissait dans la vision chrétienne comme l'histoire du salut, est devenue tout d'abord la recherche d'une condition de perfection intra-mondaine, puis, peu à peu, celle du progrès : mais la valeur finale du progrès étant de réaliser des conditions qui permettront à un nouveau progrès d'être toujours possible, son idéal se révèle vide. Le "ce vers quoi" une fois disparu, la sécularisation devient cette dissolution de l'idée même de progrès qui s'opère très précisément dans la culture des $\mathrm{XIX}^{\mathrm{e}}$ et $\mathrm{XX}^{\mathrm{e}}$ siècles. $»^{17}$ aujourd'hui ? Sociologiquement, la sécularisation des sociétés modernes a marginalisé le christianisme. Pour un observateur, l'aspect moderne de l'Eglise catholique n'est-t-il pas devenu très médiatique, voir théâtral ? Assistons-nous à une crise profonde, une mutation, ou un déclin du christianisme occidental? A ce sujet, Michel de Certeau remarque :

« Si le christianisme se réduisait à un silence, cela signifierait qu'il est mort. Seuls les morts ne parlent plus. Se contenter d'une apologie du silence, ce serait donc pratiquer un rituel d'enterrement. L'expérience chrétienne n'existe que si elle s'inscrit dans des pratiques et dans un langage. Nous assistons aujourd'hui partout à un discrédit du langage. Les signifiants se développent mécaniquement, ils saturent l'atmosphère, ils prolifèrent. Ils ne désignent plus des convictions, mais la logique de leur production. $»^{18}$

Le christianisme, sous la bannière de la civilisation occidentale, a franchi des tutelles nationales en mers et en terres: les Croisades, les Inquisitions, les méthodes d'évangélisations violentes dans les terres conquises en Afrique et en Amérique latine. Il était allé jusqu'en Chine, en se réclamant toujours de son "message universel». Il a montré, pendant deux millénaires sa capacité d'adaptation à des cultures différentes et à des civilisations différentes. Par exemple, le calendrier d'origine chrétienne adopté à la fois par l'Occident et par l'Orient n'est qu'un symbole, mais il illustre bien cette capacité d'adaptation comme "mondialisation ». A cet égard, J. Derrida remarque que :

«Il faudrait donc interroger de ce point de vue ce qu'on appelle la mondialisation et ce que j'ai proposé ailleurs de surnommer la mondialatinisation - pour prendre en compte l'effet de chrétienté romaine qui surdétermine aujourd'hui tout le langage du droit, de la politique, et même l'interprétation du dit "retour du religieux". »"

Dans la perspective de J. Derrida, le propre du langage de cet universalisme chrétien et romain est caractérisé surtout par ses mots clefs, dont le plus important : Confession, qui peut être considéré à plusieurs égard actuel, car il est lié aussi au "pardon", à une interprétation chrétienne (directe ou indirecte) du "prochain" ou du "semblable". Sur ce propos, il ajoute ainsi :

«"La mondialisation" du pardon ressemble à une immense scène de confession en cours, donc à une convulsion-conversion-confession virtuellement chrétienne, un processus de christianisation qui n'a plus besoin de l'Eglise chrétienne. $»^{20}$

Nous trouvons ces mots stratégiques de confession et de conversion au fondement des Confessions d'Augustin, entendues comme critique de soi. Chez lui, l'originalité de cette pratique consiste à traduire dans le langage philosophique, son expérience spirituelle. Augustin s'est montré penseur original et novateur du sens, au moment où s'écroule l'Empire romain, c'est-à-dire à la fin d'une époque et au commencement d'une autre, dans un " vide de sens ", résultat d'une angoisse devant l'incertain.

Le dire confessionnel trouve son modèle fondateur chez Augustin. Cette tradition subit toute une série d'évolutions et rencontre des paradoxes essentiels qui constituent 
comme les moteurs de ces évolutions successives. En effet, dans la visée d'Augustin, en l'absence d'un confesseur (institution qui sera mise en place plus tard dans l'Eglise), dire soi-même dans une entreprise confessionnelle peut devenir une redite solipsiste d'une image de soi à l'infini, et elle restera comme image tant que le sujet ne cherche pas un point d'ancrage en dehors de lui-même. Dans cet exercice, nous admettons que le confesseur ne sait rien de lui-même; il s'affiche ignorant pour se connaître. Ici un problème se pose inévitablement : comment sortir du solipsisme, autrement que par l'intervention d'une institution religieuse dont la fonction est la normalisation du dire confessionnel par l'intervention d'un intermédiaire dans la personne du confesseur? Cet effort viendra du sujet même pour briser le cercle solipsiste. Aussi, la confessionconversion devient-elle un exercice éminemment philosophique, non seulement parce qu'il existe une tradition confessionnelle attestée dans la philosophie occidentale depuis Augustin, mais aussi parce qu'il s'agit plus précisément de concevoir la philosophie comme une manière de vivre et non comme souci de construire un système. Ici, retenons ceci : ces trois notions de souci de soi, de confession et de conversion représentent une matrice stratégique pour notre sujet. Le passage du souci de soi à la confession, ainsi que la synthèse opérée par Tolstoï entre ces deux moments constituent le cœur de notre investigation.

21 Avançons avec Foucault dans le thème de la conversion dont nous avons souligné l'importance. Dans «l'herméneutique du sujet », selon Foucault :

«Il faut se rappeler que la règle d'avoir à se connaître soi-même, a été régulièrement associée au thème du souci de soi. D'un bout à l'autre de la culture antique, il est facile de trouver des témoignages de l'importance accordée au "souci de soi" et de ses connexions avec le thème de la connaissance de soi. $»^{21}$

Foucault, pour illustrer ce thème, envisage les deux extrêmes que sont d'un côté Socrate et de l'autre Grégoire de Nysse et il souligne combien la notion d'epimelia heautou les réunit : « Entre ces deux repères extrêmes (...) on peut constater que le souci de soi a constitué non seulement un principe, mais une pratique constante. $\aleph^{22}$ Dans l'optique foucaldienne, le souci de soi (cura sui) imprègne l'antiquité, le Gnôthi seauton en est une forme essentielle et non pas une exception. C'est en quoi la perspective de l'homme antique est définie à la fois par un regard sur soi-même et par une activité en accord avec cette connaissance. Ainsi, s'accomplit la plénitude de soi :

«Le terme même d'epimeleïa ne désigne pas simplement une attitude de conscience ou une forme d'attention qu'on porterait sur soi-même; il désigne une occupation réglée, un travail avec ses procédés et ses objectifs. ${ }^{23}$

Il rappelle notamment que Xénophon employait le mot "epimeleïa " pour désigner le travail de maître de maison qui dirige son exploitation agricole ${ }^{24}$ : Le sujet donc est porté par l'epimeleia, en se connaissant, il se retrouve soi-même. Mais cette retrouvaille implique qu'on se soit préalablement cherché. S'occuper de soi « c'est une forme de vie $»^{25}$, et dans cette forme de vie, le soi devient son propre objet : « De là l'idée de la conversion (ad se convertere), l'idée de tout un mouvement de l'existence par lequel on fait retour sur soi-même (eis heauton epistrepheïn). » ${ }^{26}$

De Socrate aux cyniques et aux stoïciens, l'exercice philosophique consiste en une manière de vivre qu'on peut définir comme le souci de soi et une culture de soi : une pratique de l'amitié, de l'amour, du plaisir sexuel, du soin du corps, de l'exercice de liberté, du souci des autres, du souci de vérité, bref tout ce qui touche à la vie quotidienne. Cette culture représente le monde éthique. Foucault confronte, dans son vaste travail d'investigation, les textes grecs et latins des deux premiers siècles de 
notre ère à l'époque moderne. Il remonte de la période romaine jusqu'à l'Antiquité grecque et gréco-romaine, afin d'analyser, à travers des textes pour la plupart «prescriptifs » de philosophes, de moralistes et de médecins, les problématisations morales des activités et des plaisirs sexuels et leurs rapports avec des «arts de l'existence ». L'antiquité devient une référence pour notre modernité. Ainsi, Foucault a voulu montrer que cette tradition antique a subi un changement avec l'émergence de la culture chrétienne : il s'agit d'un passage de la quête d'une éthique personnelle antique à un credo chrétien qui exige une obéissance stricte aux règles communes de l'institution ecclésiastique. Avec Foucault, nous constatons l'existence d'une tradition antique (Stoïciens grecs et romains) étudiée en détail dans L'usage des plaisirs et Le souci de soi. Au vrai, le "souci de soi ", la conversion philosophique et religieuse, ont accompagné le développement de la philosophie depuis l'Antiquité grecque. Cette tradition s'oppose à cette autre tradition de confession-conversion qui commence avec la culture chrétienne; Augustin l'illustre à travers "l'homme intérieur" de ses Confessions $^{27}$. Augustin visait non seulement sa conversion, mais la conversion de la communauté par l'intercession de son Credo. Pour ce faire, il s'adresse à son Dieu: le "Je " humain s'y constitue en dialogue avec le Toi divin. Il existe chez lui, une puissance d'interrogation qui est aussi dialogue avec autrui, dont Dieu. Est-ce que cette tradition antique dont nous avons parlé, est irrémédiablement perdue pour les modernes ? M. Foucault et P. Hadot $^{28}$ essaient de montrer le contraire.

A travers notre enquête sur l'histoire des métamorphoses du dire confessionnel et notamment chez Tolstoï, nous avons souligné que la tension entre le dire confessionnel et le souci de soi de la philosophie antique ne cessent de réapparaître ${ }^{29}$. L'aboutissement de cette évolution chez Tolstoï, après avoir affronté le nihilisme moderne, permet d'ouvrir le dire confessionnel à une autre universalité. Tolstoï qui, pendant toute sa vie, s'est senti guidé par une règle de conduite religieuse - ce qui a fait de lui un penseur religieux hétérodoxe, atypique et non systémique - a eu une influence non négligeable à travers le monde comme artiste et penseur. S'il a voulu se montrer comme un novateur du sens pour son époque post-chrétienne, c'est surtout contre le nihilisme naissant; celui que Nietzsche a philosophiquement saisi et proclamé. Nous savons que, le $\mathrm{XX}^{\mathrm{e}}$ siècle a fait de ce concept un usage déconcertant : il l'a conçu comme signe de vide. Or, Tolstoï emprunte au genre confessionnel issu d'Augustin, une certaine force génératrice contre le sens nihiliste de son époque et une impulsion en rappelant la philosophie à sa tâche, qui est moins de reconstruire un système que d'observer et de vivre : vivre et s'éprouver soi-même. En ce sens, la lecture de Tolstoï peut accompagner l'homme moderne en quête de sens, dans l'angoisse et l'incertitude, contre toute logique nihiliste. Sa pensée naît de la condition humaine, par une œuvre poursuivie sans relâche au cours d'une vie entière. Elle nous invite à faire rupture totale avec la manière habituelle de vivre et de croire, en cherchant à adhérer à une nouvelle manière de vivre. Dans notre démarche, nous situons Tolstoï dans le sillage confessionnel du christianisme dont le modèle est Augustin. Tolstoï est cette figure singulière qui affronte le nihilisme de la modernité en opérant la synthèse originale de l'exercice philosophie antique et de la confession chrétienne. Cet effort prend de multiples formes dans les productions littéraires et philosophiques de Tolstoï.

Nous pouvons, en effet, suivre l'évolution de Tolstoï à travers ses Journaux quotidiens, où il s'exerçait à "être comme il faut "; il s'agissait d'une sorte de discours thérapeutique intériorisé, signe permanent d'un immense effort sur soi-même qui dure toute une vie. Ce travail sur soi n'évacue pas le tragique: comme lui-même, ses 
personnages romanesques n'arrivent jamais à chasser le tragique. Chez lui, l'action tragique (souvenons-nous les guerres napoléoniennes dans Guerre et Paix), conduit à une libération, à une "délivrance de l'âme "; l'homme l'intérieur cherche à ne pas être le sujet de ses tourments. Tolstoï, en faisant le récit de sa vie, fait savoir à autrui comment il "corrige " son moi, en effectuant un effort actif sur sa vie, en vue du dépassement de soi. Son effort exige d'être dépassé. Il s'agit là de la faculté de s'examiner soi-même avec objectivité, en raisonnant, ce qui dépend uniquement de l'homme. La philosophie religieuse de Tolstoï extériorise ce besoin de se convertir et de convertir autrui. Tolstoï voulait trouver l'homme universel en lui-même : son Moi s'ouvre à l'universalité dans sa quête du "sens de la vie ». Chercher le sens de la vie nécessite un paradigme universel commun qui représente la vie de l'humanité sans exclusion : « il fallait chercher ce sens (le sens de la vie) (...) chez cette multitude d'êtres humains, qui ont vécu et vivent, qui organisent et supportent et leur vie et la nôtre. $»^{30}$ Tolstoï cherchait des valeurs ni dans l'histoire, ni dans sa propre culture, et ni uniquement dans la religion, mais dans l'expérience propre de l'individu. Son paradigme de l'homme comporte plusieurs cultures. Son universalisme comporte aussi bien l'Orient que l'Occident. L'influence de sa pensée sur l'idéologie de la «nonrésistance» de Gandhi aux Indes, témoigne de cet universalisme. La synthèse tolstoïenne que notre enquête a pour objectif de définir, s'oppose à la tradition du dire confessionnel, dans la mesure où celui-ci a pour origine une rupture intervenue entre le monde antique et chrétien. Cette rupture est également un moment d'indistinction: l'extérieur et l'intérieur, l'individu et la société etc. L'œuvre de Tolstoï s'inscrit dans un moment de séparation et de distinction de ces différentes sphères.

Tolstoï avait une interprétation très personnelle de la religion. Il avait posé le thème religieux comme un problème rationnel, pour affirmer le sens religieux de la vie. Il va jusqu'à réécrire les Évangiles en dehors des traditions judéo-chrétiennes. Tolstoï rejette totalement le christianisme des pères de l'Église. Il va jusqu'à réécrire les Évangiles, en « rationalisant » le contenu. Tolstoï apparaît comme un penseur post-chrétien. Il sera excommunié par l'Eglise orthodoxe. Dans la dernière partie de sa vie, étant proche des idées anarchistes, il prend ses distances avec sa vision chrétienne, et sa religiosité devient hétéroclite : Jésus, Tao, Lao-Tseu, Confucius y cohabitent ensemble et relient l'Occident et l'Orient. Il n'était certainement pas un chrétien comme Dostoïevski. A propos du nihilisme, le contraste entre Dostoïevski et Tolstoï peut éclaircir l'évolution religieuse de ce dernier. Par exemple, pour Berdiaeff :

«A l'encontre d'une opinion très répandue, on ne saurait assez énergiquement répéter que l'esprit de Dostoïevski avait une tendance à construire et non à détruire, que son état d'âme le poussait à l'affirmation, non à la négation. Mais il concevait Dieu, l'homme et le monde à travers toutes les angoisses du dédoublement et des ténèbres. S'il a jusqu'au fond compris la nature du nihilisme russe, lui-même était antinihiliste. C'est ce qui le distingue de Léon Tolstoï, que la contagion nihiliste avait gagné. $»^{31}$

Ici, il faut ajouter au jugement de Berdiaeff que, Tolstoï, au-delà de son "nihilisme » et de son moralisme, son expérience religieuse doit être étudiée dans le problème du nihilisme de l'époque, philosophiquement proclamé par Nietzsche, par «la mort de Dieu ». En dehors de la Russie, l'influence de Tolstoï fut énorme sur son époque. R. Zapata souligne que :

«L'influence de Tolstoï sur la pensée russe fut limitée car, comme ce fut le cas pour Dostoïevski, il buta sur la persistance des divisions entre "réactionnaires" 
et "progressistes" des années $60 \mathrm{du}$ XIX siècle. Mais il faut souligner l'influence majeure qu'il aura sur Gandhi et sur la pensée européenne dans son ensemble : des philosophes comme Wittgenstein et Horkheimer en ce qui concerne la recherche de fondements de l'éthique individuelle et de la religiosité. $»^{32}$ tradition issue de Saint-Augustin, se dessine un antagonisme qui a pour objet le rapport à l'histoire. En effet, dans La Cité de Dieu, Augustin, proposant une réfutation très fine de la vision du monde antique, développe une interprétation chrétienne de l'histoire et tourne son regard vers l'avenir, horizon temporel d'un but défini et d'un ultime accomplissement. A propos de son immense influence posthumes, H.-I. Marrou constate que Augustin fait une première tentative d'historicisation théologique du temps historique, où il $\mathrm{y}$ a une articulation possible de la théologie de l'histoire avec l'historiographie :

" Jusqu'à Nietzsche, toute la pensée occidentale a vécu, en ce qui concerne l'histoire, sur des schèmes chrétiens : telle qu'elle s'est développée, surtout à partir de Condorcet et de Hegel, la philosophie de l'histoire apparaît comme une transposition sur le plan naturel des concepts de base hérités de la théologie chrétienne, tel que le moyen âge l'avait hérité de saint Augustin. » $^{33}$

En revanche, chez Tolstoï, à travers sa quête religieuse, nous constaterons cette préoccupation : réfléchir profondément sur le sens de l'histoire. Pour lui, l'avenir n'est pas eschatologique. L'histoire n'est pas guidée par la Providence et n'a aucune finalité. Il n'y a ni le temps sacré, ni le temps profane. Avec une force intransigeante, Tolstö̈ voulait convaincre ses lecteurs que les hommes ne se cachent jamais derrière les évènements. A la différence des historiens, en décrivant les grands événements, réduire les acteurs historiques à l'échelle humaine ordinaire, était son but d'artiste :

«L'historien est parfois obligé, gauchissant la vérité, de ramener toutes les actions du personnage historique à l'idée qu'il a mise en lui, et à elle seule. L'artiste, au contraire, voit dans cette réduction à une idée unique une contradiction avec sa tâche et s'efforce seulement de comprendre et de montrer non pas un acteur de l'histoire, mais un homme. ${ }^{34}$

Chez Tolstoï, les temps historiques deviennent l'objet d'une critique, notamment dans Guerre et Paix, où sa poétique expose une vision philosophie et religieuse du monde. Dans ce roman bien particulier, l'écrivain livre au lecteur ses considérations philosophiques sur le sens de l'histoire en épiloguant et en passant outre les formes conventionnelles. En procédant ainsi, il rend clair le caractère confessionnel de son roman : "Qu'est-ce que la Guerre et la Paix? Ce n'est pas un roman, encore moins un poème, et encore moins une chronique historique. La Guerre et la Paix est ce que l'auteur a voulu et pu exprimer dans la forme où cela s'est exprimé. $»^{35}$ Dans sa critique contre l'historicisme ambiant de son époque, Tolstoï n'attribuait ni sens, ni espérance à la finalité historique. Chez lui, le temps historique devient l'objet d'une quête personnelle et religieuse, durant les guerres napoléoniennes, dans Guerre et Paix, le personnage romanesque (Pierre) qui est le porte-parole de Tolstoï, s'interroge sur « le sens de la vie ", il est toujours dans le temps réel et n'évacue jamais la cruelle réalité des événements, il ne cherche pas un salut eschatologique. L'intérêt de Guerre et Paix réside dans ce fait que, l'écriture de l'histoire est à jamais une entreprise incertaine. La critique de l'historicisme s'inscrit au cœur de notre propos: de la grâce, de l'historicisme, de l'universalisme occidental... 

discouru sa vie durant, est indissociable de ce qu'il a vécu. Son écriture fonctionne comme un port parole de ses expériences vécus complexes. Dans ses œuvres littéraires, le «je " tolstoïen est identifiable et il nous renvoie à un certain «pacte autobiographique » entre l'auteur et son œuvre. Ce "pacte autobiographique $»^{36}$ est une caractéristique structurante dans sa créativité littéraire. Chez l'écrivant, le pacte autobiographique est une œuvre d'art par excellence: travailler de manière originale le langage du récit pour construire un chemin exigent et authentique vers "la vérité ", pour pouvoir s'adresser à l'inconnu. A ce sujet, rappelons avec P. Lejeune que l'autobiographie constitue l'une des formes les plus complexes dans la littérature occidentale. Ecrire en prenant sa propre personne comme sujet est une activité littéraire particulière qui pose bien des questions. Car, la mémoire est sélective, et, à partir du moment où elle commence à se constituer, elle procède à un tri et ne garde que des fragments et des phases décisives de notre passé. Nul ne peut prétendre se souvenir de la totalité de sa vie. Sur ce «pacte autobiographique » entre l'auteur et son œuvre, Roland Barthe est un autre exemple connu : Roland Barthe par Roland Barthe. Ce livre montre un tournant important dans ses œuvres. Car, il s'agit plutôt d'un Barthe qui ne se réfère pas à des appuis «scientifiques" auxquels il avait jusqu'alors recours (la linguistique, la psychanalyse...). Au lieu d'un discours construit de la dissertation pour un texte fragmenté, le sujet-écrivain parle de lui avec une certaine distance constante et ironie : il le fait se désigner tour à tour sous la forme du « je », du « il », du « vous » ou de ses initiales « R.B. », et finalement dit « je » :

" ... Et quelquefois, dérision de tout ceci, "il" cède la place au "je" sous le simple effet d'un embarras syntaxique: car dans une phrase en peu longue, "il" peut renvoyer sans prévenir à bien d'autres référents que moi. ${ }^{37}$ un personnage du roman. »

Expliquons-nous davantage pour mieux saisir l'idée structurante de l'entreprise confessionnelle de Tolstoï : sa quête de soi est représentée par une pensée religieuse prédicative et hétéroclite, par une culture philosophique autodidacte. Il est l'un des écrivains rares qui ait réussi à concilier la profondeur et l'érudition. Sa quête prend son sens autour d'une préoccupation existentielle bien précise : la mort, c'est-à-dire ce que la vie a inventé comme réalité la plus ultime. Chez lui, le lecteur est frappé par une sorte de l'enivrement de l'écrivain pour le fait de mourir, où il puise sa force créatrice. Dans sa pensée, cette idée vise à permettre à l'homme d'assumer sa finitude par un effort sur-soi en interrogant la finitude au quotidien. Tolstoï écrivain fait de la mort une puissance pédagogique notamment à travers les récits comme La mort d'Ivan Ilitch et Maître et Serviteur, pour "dévoiler" le sens de sa finitude à autrui, pour que son "message" devienne "contagieux", pour qu'autrui fasse cet effort sur-soi et s'arrache ainsi à l'ordre répétitif du quotidien. Dans une période plus cruciale de sa vie, au sommet de sa force créatrice, Tolstoï, en faisant une sorte de bilan de sa vie désenchantée, écrit un texte étrange: Confession (1879), où il se demande constamment : «Y a -t-il dans ma vie un sens tel qu'il ne soit pas anéanti par la mort qui m'attend inévitablement ? " ${ }^{38}$ En effet, «Que faire?», "A quoi bon de vivre?», sont des questions qui hantent Tolstoï. Il frôle le suicide. Tolstoï souffre par ce que sa vie n'a pas de sens à laquelle il cherche un sens. A travers ces questions existentielles répétitives qui travaillent toute sa vie et son œuvre, Tolstoï voulait embrasser la condition humaine. Avec toute la rigueur de sa créativité littéraire, il a transformé 
l'idée de la mort en une puissance d'interrogation de soi-même comme un appel à autrui. Sa philosophie comme manière de vivre et ses paradoxes sont liés à cette quête. Cette interrogation radicale et existentielle faite sur soi et sur autrui l'illustre par excellence. Dans cette quête, l'idée de la mort exhorte chacun à briser la routine de la vie quotidienne, elle met le sujet face à l'obligation de se détacher de toute réflexion qui ne résulterait pas de la quête de soi. Le sujet sera engagé dans ce qui sera pour lui peut-être la "dernière occasion ", pour progresser dans cette quête qui nécessite une manière de vivre authentique : c'est ici que philosopher devient une manière de vivre. A ce sujet, il faut se rappeler que Heidegger, s'intéressait au récit de Tolstoï, La Mort d'Ivan Ilitch. Pour le philosophe allemand, la société des hommes tend à masquer la réalité de l'être pour la mort, qui est le cas dans le récit de Tolstoï et il cite en guise d'illustration La Mort d'Ivan Ilitch: «L. N. Tolstoï a décrit dans son récit La mort d'Ivan Ilitch, le phénomène de ce "on meurt" qui bouleverse tout et où tout s'effondre. $»^{39} \mathrm{Par}$ cette référence, Heidegger a voulu montrer que la mort d'Ivan Ilitch fait éclater la révélation de ce douloureux arrachement à la quotidienneté du "on" et toute l'hypocrisie de son entourage. Car, dans le récit, le souci de quotidienneté ne suffit pas à gommer la mort comme réalité dans l'oubli : «Personne n'osait, tous craignaient que soudain le mensonge correct ne soit détruit, et que la réalité ne devienne évidente à tous les yeux. $»^{40}$ L'énigme qui semble dominer tout le texte consiste à savoir comment on passe du « on » de la banalité et de l'indifférence à la réalité de la mort, qui est la mort Ivan Ilitch. Et en ce sens, l'originalité radicale de ce récit de Tolstoï consiste à faire de la mort "d'un homme ordinaire " un événement extraordinaire. Ainsi, l'habile pédagogue fait de la mort une puissance pédagogique pour dévoiler le sens de sa finitude à autrui, pour que son "message » devienne "contagieux ", pour qu'autrui s'exhorte soi lui-même et qu'il soit arraché à l'ordre répétitif du quotidien. L'entourage du mourant a une attitude "non authentique» et "escamote» ainsi la mort. Heidegger, dans son livre l'Etre et le Temps écrit que :

«La vie publique où prend place l'être en compagnie quotidienne "connaît" la mort comme une rencontre qui se produit constamment, comme "cas de mort". Un tel, qu'il soit proche ou lointain, "meurt".., "La mort", poursuit Heidegger, en tant que telle, se maintient dans l'insurprenance qui caractérise ce qui se rencontre quotidiennement. Le on s'est déjà assuré aussi pour cette événement d'une explication. Les propos tenus à son sujet, qu'ils soient clairement exprimés ou le plus souvent restreints à de "fugitives" allusions, reviennent à dire : on finit bien un jour par mourir mais pour le moment nous-on demeure à l'abri. $»^{41}$

Pour Tolstoï, assumer la finitude demande un effort quotidien, la maîtrise de soi, l'appréciation exacte des limites du vrai, la mesure et la force.

Par delà le nihilisme et l'histoire chrétienne de la confession, Tolstoï retrouve avec originalité une tradition issue de l'antiquité et opère une synthèse tout à fait spécifique. Chez lui, la quête du sens mobilise toutes les ressources des traditions du dire confessionnel et du souci de soi. Il nous arrive, à nous modernes, assez souvent, même si ce n'est pas tous les jours, de nous demander si la vie a un sens et en quoi elle consiste. Dans cette perspective, la quête de sens peut devenir l'objet d'une quête de soi, face à une ultime vérité de la vie : la mort. Nous les modernes, nous savons aussi que, le « désenchantement » weberien a ruiné « la paix intérieure » de l'homme; paix dans laquelle il était supposé vivre auparavant sa spiritualité. La fameuse déclaration de Nietzsche : «La mort de Dieu » exprime la même inquiétude. Pour notre sujet, cette quête est le récit d'une vie, le récit confessionnel de Tolstoï. Cet effort comporte un certain but qui se manifeste comme une orientation vers le «Bien ». Face à la finitude 
inévitable de l'homme, chercher ce "Bien» comme sens de la vie constitue une narration cohérente dans son entreprise confessionnelle. Seul l'effort d'une expérience personnelle pourrait faire avancer l'individu dans cette orientation. Le sujet perçoit sa vie comme une quête et dans la mesure où il prend du recul par rapport à soi-même, il s'oriente vers ce qu'il n'est pas encore. Dans cette expérience, l'individu pourrait se connaître à travers ses échecs, ses réussites et ses progrès, sans jamais devenir un ascète-automate. Il essaie de voir le sens de sa vie au-delà de sa mort : c'est l'effort sur soi. C'est cette quête qui résume l'interrogation tolstoïenne de soi et de l'autre ; elle est au centre de cette pensée religieuse hétérodoxe et atypique, elle réactualise ainsi le " problème du sens » qui nous permettrait aussi d'essayer de réactualiser une certaine manière de philosopher qui consiste à vivre. Tolstoï est moderne. Son entreprise a pris durant toute sa vie des formes différentes : romans, récits, essais, confession, journal intime, pensée philosophique, pièce de théâtre, où sa créativité littéraire se montre d'une manière originale. Avec Rousseau, Nietzsche, Kierkegaard, nous pouvons inscrire Tolstoï dans la tradition de la "critique dénonciatrice de soi ", dans le sillage augustinien. Ces philosophes n'avaient pas le souci de fonder un système philosophique pour le professer ni de bâtir une école de leur vivant. Philosopher pour eux, était d'abord une manière de vivre, exigée par leur quête intérieure. Si Rousseau, Nietzsche et Kierkegaard représentent les grands moments de dire confessionnel après Augustin, pour nous Tolstoï peut être considéré comme le dernier exemple original après eux. Nous savons que Tolstoï a eu une influence énorme sur la littérature et la pensée occidentale. S'il peut être considéré contemporain de Nietzsche et de Kierkegaard, il connaissait superficiellement le premier et probablement pas le deuxième. Tolstoï le religieux avait certaines affinités aussi bien avec Nietzsche qu'avec Kierkegaard.

\section{NOTES}

1. Voir Mehmet Aydın, Saint Augustin et Léon Tolstoï: confesser en philosophant? thèse de philosophie soutenue sous la direction d'Alain Brossat, Paris VIII, 2003.

2. Friedrich Nietzsche, Fragments postumes (automne-hiver 1872), cité in Jean Noël Vuarnet, Le philosophe-artiste, Paris, UGE, coll. « 10/18 » 1977, p. 104.

3. Voir V. Descombes, Proust. Philosophie du roman, Minuit, 1987, pp. 88-90.

4. T. Mann, Goethe et Tolstoï, Editions Victor Attinger, Collection Occident, Paris, 1947, p.19- 20.

5. Dictionnaire de la foi catholique, 1986, Editions C.L.D. Dom Guy- Marie Oury Moine de Solesmes, p.5I.

6. M. Foucault, Volonté de savoir 1, Histoire de la sexualité, Gallimard, 1976, pp. 27-28.

7. J. Delumeau L'aveu et le pardon, Les difficultés de la confession XIII ${ }^{\mathrm{e}}-\mathrm{XVIII}^{\mathrm{e}}$ siècles, Fayard, 1990, p. 12.

8. Ibid., pp. 13-14.

9. J.-C. Eslin, Saint Augustin L'homme occidental, Michalon, 2002, Paris, pp. 13-14.

10. Ibid., p. 10.

11. Voir K. Jaspers, Les grands philosophes, 2, « Saint Augustin », traduction française, traduit par G. Floquet, Agora, Plon, p. 305. 
12. Voir K. Jaspers, « Parallèle avec Kierkegaard et Nietzsche », ibid.., pp. 265-268.

13. Voir Isabelle Bochet, Introduction, Augustin dans la pensée de Paul Ricoeur, 2003, Editions Facultés jésuite de Paris.

14. J.-F. Lyotard, La confession d'Augustin, Galilée, Paris, 1998, p. 91.

15. J. Guitton, Actualité de Saint Augustin, Grasset, 1955, p. 14.

16. P. Courcelle, Les Confessions de Saint Augustin dans la tradition littéraire, 1963, Paris.

17. G. Vattimo, Critique de la modernité, Nihilisme et herméneutique dans la culture postmoderne, Seuil, traduction italienne, 1987, p.13-14..

18. M. de Certeau, J.-M. Domenach, Le Christianisme éclaté, Seuil, 1974, pp. 73-74.

19. Foi et Savoir de J.Derrida suivi de Le Siècle et le Pardon entretien avec M.Wieviorka, Seuil, Coll. Point Essais, 2001, p. 108.

20. Ibid., p. 107.

21. M. Foucault, Résumé des cours, Juliard, 1970-1989, Paris, 1989, p. 145.

22. Ibid., p. 147.

23. Ibid., p. 148.

24. Ibid., p. 148.

25. Ibid., p. 150.

26. Ibid, p. 150

27. Saint Augustin, Les Confessions (S. AURELI AUGUSTINI CONFESSIONES). La traduction de P. Cambronne, Pléiade, éd. Gallimard, 1998. Edition publiée sous la direction de Lucien Jerphagnon : les Confessions et Dialogues philosophiques, CEuvres, I.

28. P. Hadot, Qu'est-ce que la philosophie antique? Gallimard, 1995.

29. Voir Mehmet Aydın, Saint Augustin et Léon Tolstoï: confesser en philosophant? thèse de philosophie soutenue sous la direction d'Alain Brossat, Paris VIII, 2003.

30. L. Tolstoï, Confession, la traduction de L. Jurgenson, Pygmalion, Gérard Watelet, 1998, p. 67.

31. Nicolas Berdiaeff, L'esprit de Dostoïevski, traduit du russe par Alexis Neville, 1974, Stock, p. 39-40.

32. Voir R.Zapata, "Tolstoï ", La philosophie russe et soviétique, que sais-je, 1988, p.56 ; voir aussi J. Bouveresse, Wittgenstein : la rime et la raison, les éditions de minuit, 1971, p. 80-1.

33. H.-I. Marrou, L'ambivalence de l'histoire chez saint Augustin, Paris, Vrin, 1950, p.15.

34. Cité par Michel Aucouturier, Tolstoï, Seuil, 1996, Paris, p. 84.

35. L. Tolstoï, Guerre et Paix, (Appendice), Pléiade, p. 1611.

36. Voir P. Lejeune, Le pacte autobiographique, Seuil, Paris, 1996.

37. Roland Barthe par Roland Barthe, 1995, Seuil, p. 147.

38. L. Tolstoï, Confession, op. cit., p.40.

39. Heidegger, L'être et le Temps, trad. par F.Vezin, Gallimard,1986, p. 308.

40. L.Tolstoï, La Mort d'Ivan Ilitch, nouvelles et récits (1851-1885), Flammarion, coll. « GF », traduit par M. Cadot, 1993. p. 384.

41. Heidegger, L'être et le Temps, op. cit., p. 307.

\section{RÉSUMÉS}

Romancier, conteur, essayiste, pédagogue, prédicateur, penseur politique et religieux, sociologue, philosophe, Tolstoï est une des figures les plus marquantes de la littérature russe et 
mondiale. Le message universel de son oeuvre se présente sous des facettes multiples. Nous avons étudié Tolstoï du point de vue philosophique. Notre propos n'est donc ni littéraire, ni artistique, ni même à proprement parler religieux. C'est à la frontière de toutes ses disciplines que la philosophie entendue comme exercice nous permettra de circuler afin de mettre en évidence l'aspect vivant de cet exercice, tel qu'il est pratiqué aussi par Tolstoï. Le style confessionnel apparaît dans ce cadre comme la clef de l'originalité de l'œuvre tolstoïenne. La confession dans ses relations contradictoires avec la philosophie identifierait l'Occident. Par delà l'abîme historique qui sépare les deux penseurs, Saint-Augustin est un passage obligé pour comprendre la démarche tolstoïenne. Tolstoï emprunte le genre confessionnel issu d'Augustin.

INDEX

Mots-clés : confession, connaissance de soi, philosophie 